\title{
Scalp metastases as the first sign of a breast carcinoma
}

\author{
Magdalena Ciążyńska', Katarzyna Płużańska-Srebrzyńska², Joanna Narbutt², Paweł Kolano³, \\ Olga Stasikowska-Kanicka ${ }^{4}$, Aleksandra Lesiak ${ }^{2}$

\begin{abstract}
${ }^{1}$ Department of Proliferative Diseases, Nicolaus Copernicus Multidisciplinary Centre for Oncology and Traumatology, Lodz, Poland ${ }^{2}$ Department of Dermatology, Paediatric Dermatology and Dermatological Oncology, Medical University of Lodz, Lodz, Poland ${ }^{3}$ Department of General and Oncological Surgery, Tomaszow Health Centre, Tomaszow Mazowiecki, Poland ${ }^{4}$ Department of Nephropathology, Medical University of Lodz, Lodz, Poland
\end{abstract}

Adv Dermatol Allergol 2021; XXXVIII (3): 530-532 DOI: https://doi.org/10.5114/ada.2021.107942

Cutaneous metastases (CM) are relatively uncommon and occur in $0.7-10.4 \%$ of all patients with diagnosed tumour [1]. Overall the most common malignancies that give rise to cutaneous metastases are melanomas, followed by breast cancers in females and lung cancers in males. In most of cases, the presence of cutaneous metastases is associated with advanced systemic disease, where the primary cancer is widespread and is related to poor prognosis [2, 3]. Above $30 \%$ of skin metastases are diagnosed as the first symptom of an internal cancer, where the localization and histological examination of metastasis may be an indication of the tumour origin [1]. The wall of the chest and abdomen are the most common sites of breast cancers skin metastases, but due to a high rate of this cancer, dermatologists and oncologists should remember that the lesions can also occur at the extremities as well as in the head and neck region [2].

In clinical practice, nodules are the most common presentation of cutaneous metastases of breast cancer, but a wide range of clinical manifestations are described [2]. Therefore, differential diagnostics of cutaneous metastases suggestive of breast cancer are a challenge for the dermatologist, who is often the first person who may suspect this cancer or its recurrence. Early diagnosis of skin metastases often initiate treatment or dramatically change the previously used therapeutic regimens that can prolong the life of the patient. We present the rare case of cutaneous metastases which mimic mild skin changes, which are difficult to identify.

A 47-year-old woman with a history of hypothyroidism and supraventricular tachycardia who was besides in good health reported to the Dermatologic Outpatient Clinic due to a focal hair loss in the occipital area of the scalp. The lesion was $5 \mathrm{~cm}$ large, of irregular shape, pearly-pink coloured, painless and non-pruritic. The skin was palpably elevated and partially covered with yellowish scale (Figure 1). The patient firstly noticed small hair loss a couple months before the admission and it gradually became larger. She had no history of previous dermal diseases.

A punch biopsy was taken from the skin lesion which revealed metastatic adenocarcinoma. On immunohistochemistry (IHC) staining for estrogen receptors (ER, 90\%) and progesterone receptors (PR, 70\%), mammaglobin and gross cystic disease fluid protein (GCDFP) were positive, what suggests metastatic breast adenocarcinoma with HER2 negative expression and poorly differentiated, high-grade (G3) invasion. Computed tomography imaging has shown a primary tumour in the left mammary gland. The patient denied any personal or family history of prior breast or invasive ovarian cancer as well as radiation treatment. She admits she has never done breast self-examination and clinical breast examination. She has never had any breast imaging, including mammography, magnetic resonance imaging (MRI), or ultrasound imaging. The patient was referred to the Oncology Department where she was prescribed doxorubicin with cyclophosphamide chemotherapy. After six cycles, the primary lesion is under control and skin lesions have regressed but not completely disappeared (Figure 2). At the moment, she is undergoing hormonal treatment, however her hair has regrown in the alopecia lesion.

The characteristic of malignant tumours is the possibility to develop metastases that is a threat to the life of a patient and incontrovertibly indicate systemic carcinogenesis. Metastases to the skin are quite rare when compared with metastases to other organs. The early recognition of skin changes associated with an underlying systemic carcinogenesis is very important for correct prompt diagnosis and for the initiation of proper

Address for correspondence: Magdalena Ciążyńska, Department of Proliferative Diseases, Nicolaus Copernicus Multidisciplinary Centre for Oncology and Traumatology, 62 Pabianicka St, 93-513 Lodz, Poland, e-mail: ciazynska.magdalena@gmail.com Received: 19.09.2019, accepted: 11.10.2019. 


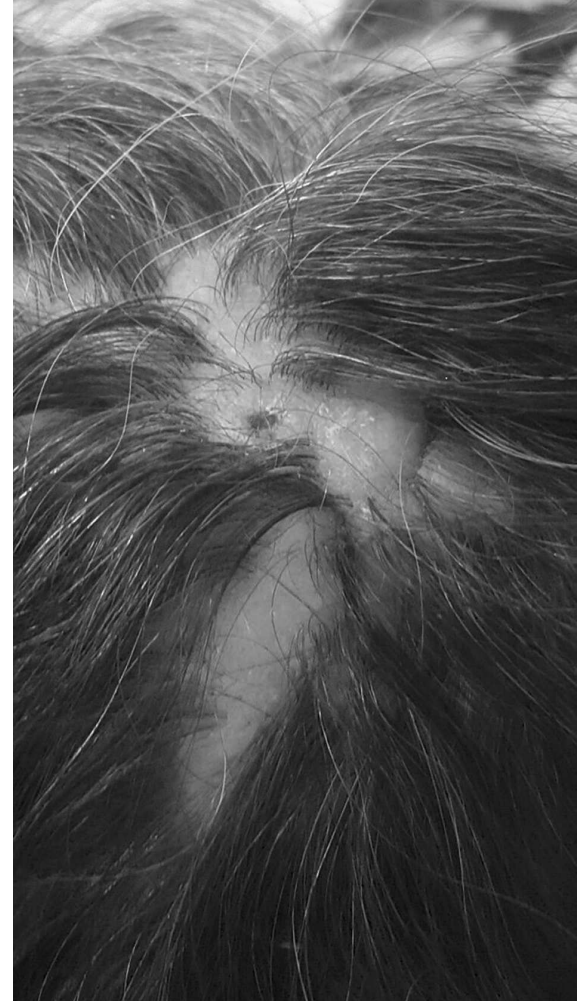

Figure 1. The asymptomatic, alopecic lesions on the scalp with central yellowish plaque scale

treatment [4]. Breast cancer is one of the most common tumours which are the leading cause of morbidity and mortality in women. A large retrospective study of 4020 patients reported that skin metastasis occurs in $23.9 \%$ of females with breast cancer [5].

However, a palpable nodule in the breast is the most common sign of breast cancer, skin lesions are the most frequent cause why women may seek for medical attention. The most frequent location of breast cancer cutaneous manifestations is the skin of the chest wall and such manifestations include inverted nipples, ulcerations, eczema-like changes on the skin or satellite nodules. Often the sign of orange peel appearance could be observed, which is the cause of the presence of cancer cells blocking the lymphatic vessels in the skin. However, distant skin metastases of breast carcinoma as the first sign of invasive tumour are quite often reported, in many cases the diagnosis of advance carcinoma was ambiguous and astonishing. The most common clinical manifestation of spreading breast cancer to the skin is the development of usually painless and firm nodules of many colours and shapes.

Alopecia neoplastica is a rare form of dermal metastases, and the scalp hair loss is secondary to dermal infiltration of tumour cells originating from a metastatic malignancy - not from the primary cancer that is occurring on the skin of the scalp. The most common clinical

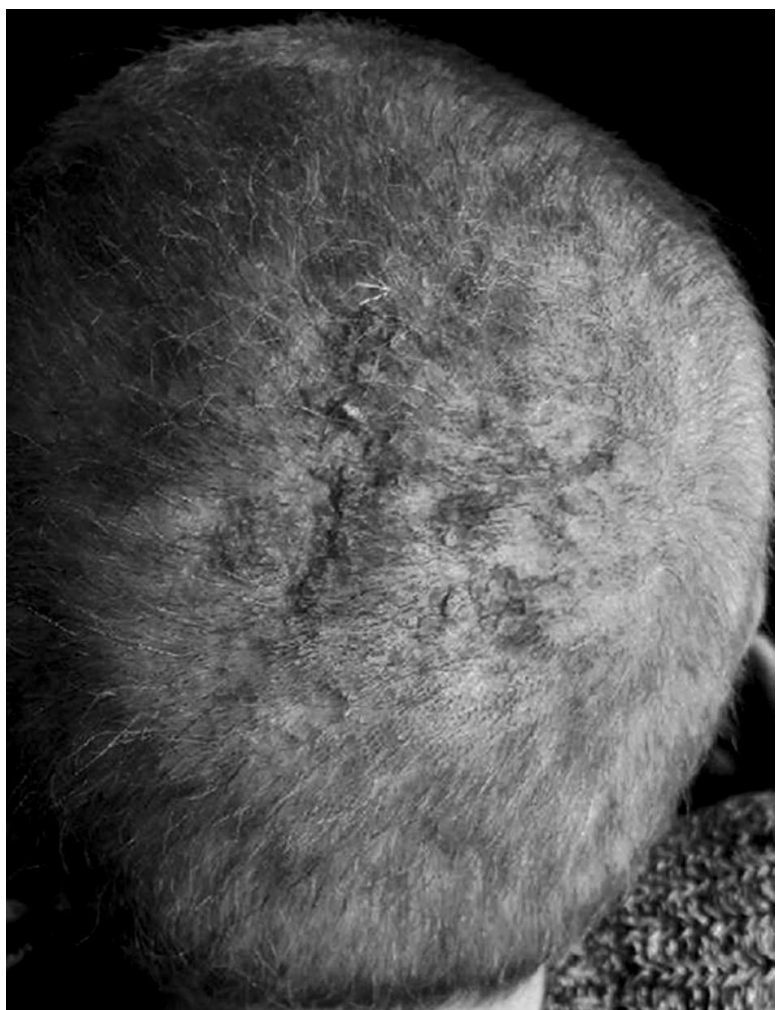

Figure 2. Regressed skin lesions after six cycles of doxorubicin with cyclophosphamide chemotherapy

manifestation of alopecia neoplastica is a single lesion - usually pink, blue or purple plaque with visible telangiectasia, where the hair loss occurs. It must be differentiated from conditions such as alopecia areata and discoid lupus erythematosus. Alopecia neoplastica is mainly caused by breast cancer [6]. Metastases may be treated locally, but in most cases the systemic treatment such as chemotherapy, radiotherapy, intratumoral chemotherapy or surgical intervention is necessary [6]. Mainly when the systemic treatment is effective with the primary tumour, it would resolve also the skin metastases.

In the presented cases it is worth emphasizing that despite the development and advanced imaging studies the patient had not previously been diagnosed for cancer and the occurrence of the skin lesion led to detection of malignancy. The attentive physical examination including skin monitoring is still irreplaceable for early detection of cutaneous metastasis. Early diagnosis of breast cancer metastasis may play a crucial role for the choice of the most effective therapeutic option. Especially in the cases when the cutaneous lesions are the first symptom of cancer. Therefore, every suspected change of unknown origin requires histopathological evaluation. The purpose of this article is to increase alertness in dermatologists and oncologists and to facilitate the recognition of the rare presentations of skin metastatic breast cancer, which is alopecia neoplastica. What is more, we present 
the effect of treatment with cytostatics, which was associated with a surprisingly good positive response also within metastases with complete hair regrowth. It is important to include this into differential diagnosis of skin lesions.

\section{Conflict of interest}

The authors declare no conflict of interest.

\section{References}

1. de Jesus Semblano Bittencourt M, Carvalho AH, Nascimento BA, et al. Cutaneous metastasis of a breast cancer diagnosed 13 years before. An Bras Dermatol 2015; 90 (3 Suppl. 1): 134-7.

2. De Giorgi V, Grazzini M, Alfaioli B, et al. Cutaneous manifestations of breast carcinoma. Dermatol Ther 2010; 23: 581-9.

3. Reich A, Kobierzycka M, Woźniak Z, et al. Keratoacanthomalike cutaneous metastasis of lung cancer: a learning point. Acta Derm Venereol 2006; 86: 459-60.

4. Rolz-Cruz G, Kim CC. Tumor invasion of the skin. Dermatol Clin 2008; 26: 89-102.

5. Lookingbill DP, Spangler N, Helm KF. Cutaneous metastases in patients with metastatic carcinoma: a retrospective study of 4020 patients. J Am Acad Dermatol 1993; 29: 228-36.

6. Cohen PR. Primary alopecia neoplastica versus secondary alopecia neoplastica: a new classification for neoplasmassociated scalp hair loss. J Cutan Pathol 2009; 36: 917-8. 